** Now published as an Open Access article in Nature Aging **

Sinclair, A.H., Stanley, M.L., Hakimi, S., Cabeza, R, Adcock, R.A., \& Samanez-Larkin, G.R. Imagining a personalized scenario selectively increases perceived risk of viral transmission for older adults. Nat Aging 1, 677-683 (2021).

https://doi.org/10.1038/s43587-021-00095-7

\title{
Imagining a Personalized Scenario Selectively Increases Perceived Risk of Viral Transmission for Older Adults
}

\begin{abstract}
Alyssa H. Sinclair ${ }^{1,2}$, Matthew L. Stanley ${ }^{1,2}$, Shabnam Hakimi ${ }^{1}$, Roberto Cabeza ${ }^{1,2}$, R. Alison Adcock ${ }^{1,2,3}$, \& Gregory R. Samanez-Larkin ${ }^{1,2}$
\end{abstract}

${ }^{1}$ Duke University, Center for Cognitive Neuroscience; ${ }^{2}$ Duke University, Department of Psychology \& Neuroscience; ${ }^{3}$ Duke University, Department of Psychiatry \& Behavioral Sciences

Corresponding Author: Alyssa H. Sinclair, ahs50@duke.edu Main Text Word Count: 3,436

Keywords: COVID-19, aging, cognition, risk perception, episodic simulation, socioemotional selectivity, memory, decision-making 


\section{Imagining a Personalized Scenario Selectively Increases Perceived Risk of Viral Transmission for Older Adults}

The COVID-19 pandemic has created a serious and prolonged public-health emergency. Older adults have been at substantially greater risk of hospitalization, ICU admission, and death due to COVID-19; as of February 2021, over 81\% of COVID-19-related deaths in the U.S. occurred for people over the age of $65^{1,2}$. Converging evidence from around the world suggests that age is the greatest risk factor for severe COVID-19 illness and for the experience of adverse health outcomes ${ }^{3,4}$. Therefore, effectively communicating health-related risk information requires tailoring interventions to older adults' needs ${ }^{5}$. Using a novel informational intervention with a nationally-representative sample of 546 U.S. residents, we found that older adults reported increased perceived risk of COVID-19 transmission after imagining a personalized scenario with social consequences. Although older adults tended to forget numerical information over time, the personalized simulations elicited increases in perceived risk that persisted over a 1-3 week delay. Overall, our results bear broad implications for communicating information about health risks to older adults, and they suggest new strategies to combat annual influenza outbreaks.

\section{Main}

News and social media have repeatedly documented the risky behaviors of Americans throughout the pandemic, and recent survey evidence suggests that Americans tend to underestimate risk related to COVID-19 transmission ${ }^{6}$. As COVID-19 has spread, so too has misinformation about both the efficacy of different preventative behaviors (e.g., mask-wearing, hand-washing) and the risks of engaging in certain commonplace behaviors where the virus could be transmitted (e.g., grocery shopping, indoor dining, air travel). Unfortunately, those most at risk of severe illness and death due to COVID-19 (i.e., older adults) are also most susceptible to believing misinformation. Older adults are far more likely to believe and share false information from social media ${ }^{7-9}$, and this problem is getting worse as increasing numbers of older adults become active on social media ${ }^{10}$.

To combat COVID-19-related misinformation and to ensure that individuals who are most at-risk for severe illness (older adults) possess the information needed to make informed decisions, it is critical to develop interventions that meet the needs of older adults by (1) effectively conveying the risks of engaging in behaviors that could cause viral transmission, and (2) ensuring that risk information sticks over time. We developed an interactive intervention that would inform individuals about COVID-19-related risks, with the intention of improving downstream compliance with public health measures ${ }^{6}$. In the present study, we tested the efficacy of our intervention across the adult lifespan and compared strategies for communicating risk information to older adults. Drawing on theoretical frameworks of aging and motivation ${ }^{11,12}$, we designed our intervention to include elements that could optimize learning for older adults. 
Past efforts to develop interventions for improving risk estimation have shown some success, but the effect sizes across interventions are typically small, and the effects rapidly diminish over relatively short delays ${ }^{13-16}$. Although older adults typically self-report being more risk averse ${ }^{17}$, their choice behavior is not always consistent with their stated preferences ${ }^{18}$. In some situations, older adults take more risks than younger adults ${ }^{19}$. Furthermore, older adults tend to seek out less information about risk $^{12}$, which can have negative consequences for their health-related decisions ${ }^{20,21}$. Older adults are more prone to deliberately choosing ignorance, especially when the new information could be negative ${ }^{22}$. These problems may also be exacerbated because older adults tend to be less successful at learning from numerical feedback ${ }^{23,24}$.

However, personalized social information may help motivate older adults to improve risk literacy. Socioemotional Selectivity Theory (SST) posits that older adults are more motivated to make decisions that maximize emotional meaning, enhance social connections, and emphasize personally-relevant factors ${ }^{11,25,26}$. Prioritizing personally-relevant social connections is adaptive when one perceives limited time left in life; bolstering social connections can offer emotional rewards and the practical benefits of a support network ${ }^{11,27}$. Importantly, these motivational changes that occur later in life correspond to broad changes in decision-making, emotion regulation, learning, and information-seeking ${ }^{11,12}$.

Leveraging these theoretical insights from SST, we predicted that if older adults are more motivated to attend to personally-relevant social information, then they may be more responsive to an intervention that involves generating rich, personalized mental imagery about close others. Past studies have used a type of mental imagery, termed episodic simulation, to enhance subsequent decision-making processes ${ }^{28}$. Converging lines of research suggest that episodic simulation of the downstream outcomes of choices can improve subsequent decision making, including self-regulation ${ }^{29-32}$. In particular, episodic simulations that involve imagining scenarios that are directly relevant for behavior ${ }^{33}$ and/or more vivid ${ }^{31}$ are most effective. Similarly, imagining a self-relevant scenario can lead individuals to judge the event as more likely to occur $^{34}$, especially if the scenario is easier to imagine ${ }^{35}$. Therefore, a personalized episodic simulation could influence beliefs about risk and enhance learning over time, particularly for older adults who are most at-risk.

In this large-scale, multi-session study, our primary objective was to develop an effective intervention to change subjective perceived risk related to COVID-19. The present study was part of a larger project; in a separate report, we describe the overall intervention results ${ }^{6}$. Here, we specifically investigate whether our intervention differentially affects younger and older adults; our approach unifies theoretical insights from past studies on episodic simulation and motivation in older adults. In the present study, we identify novel age-related differences and compare the efficacy of several strategies for communicating information about virus transmission risk. 
Our intervention involved presenting two kinds of information about risk: episodic and numerical information. We hypothesized that a personalized episodic simulation (relative to an impersonal or unrelated simulation) would facilitate subsequent learning about numerical risk information (particularly among older adults) because this task connects risk information with personally-relevant social consequences. However, we expected that older adults would be less responsive to numerical information about risk. As a secondary, exploratory objective, we also investigated whether a personalized episodic simulation would motivate further informationseeking, encouraging ongoing learning after the intervention.

We recruited a nationally-representative online sample of 545 U.S. residents (stratified by age, gender, and race to approximate the demographic makeup of the nation) (Methods, Participants). Participants completed a survey about perceived risk (due to COVID-19) of engaging in various everyday activities in their local community (e.g., grocery shopping, dining inside a restaurant) (Methods, Survey). Next, we randomly assigned participants to complete one of three variants of the episodic simulation task (Methods, Episodic Simulation Task). In the Personal simulation condition, participants imagined a scenario in which they hosted a dinner party attended by four specific close others (e.g., friends, neighbors). In this scenario, a guest became seriously ill with COVID-19, exposed the other guests to the disease, and infected the host as well. In the Impersonal simulation condition, participants imagined a fictional character experiencing the same scenario. In the Unrelated (control) condition, participants imagined a scenario that was neither personalized nor related to COVID-19. This control condition equated attention and time-on-task, but we did not expect this unrelated imagination exercise to influence subsequent learning. The episodic simulation was always the first part of the intervention, because prior studies have shown that an imagination exercise influences subsequent decisionmaking ${ }^{28,31,32}$. We expected that imagining a COVID-related scenario, especially if it was personalized, would change the way that participants engaged with subsequent information about risk probabilities.

After the episodic simulation, participants completed the second half of the intervention, which presented numerical information about risk. All participants completed a risk estimation task that involved predicting and receiving feedback about the prevalence of COVID-19 cases in their local communities (Methods, Risk Estimation Task). To quantify the strength of this numerical risk intervention, we calculated information prediction errors, the discrepancy between predicted and actual risk values. If numerical risk information drives learning, then larger prediction errors (reflecting risk misestimation) should predict larger changes in perceived risk. Finally, after the two-part intervention, participants completed the survey of perceived risk again (regarding everyday activities) (Methods, Survey). To assess the immediate and longlasting effects of the intervention, we measured perceived risk both immediately after the intervention (Session 1) and after a delay of 1-3 weeks (Session 2).

In a separate report, we described how the intervention effectively realigned perceived risk with actual risk ${ }^{6}$. Here, we tested whether the effects of the intervention differed across the 
adult lifespan. Using multiple linear regression, we predicted immediate post-intervention change in perceived risk (immediate post-intervention - baseline) from the variables age (continuous), simulation condition (Personal/Impersonal/Unrelated), average prediction error, and all interaction terms. For all statistical analyses, we report standardized beta values (and 95\% confidence intervals around the slope estimate); these measures indicate effect size. As reported elsewhere $^{6}$, we found a main effect of prediction error driving change in perceived risk $(\beta=0.22$, $95 \%$ CI $[0.14,0.31], t=5.06, p<.001)$, demonstrating that numerical feedback helped to realign perceived risk with actual risk. There was also an interaction between prediction error and simulation condition predicting change in perceived risk ${ }^{6}$, such that learning from numerical information was enhanced when it was preceded by either the Personal or Impersonal simulation (Personal vs. Unrelated: $\beta=0.16,95 \%$ CI [0.04, 0.29], $t=2.61, p=.009$, Impersonal vs. Unrelated: $\beta=0.17,95 \%$ CI $[0.05,0.29], t=2.73, p=.007$, Personal vs. Impersonal: $\beta=-0.003$, $95 \%$ CI $[-0.12,0.12], t=-0.04, p=.965)$. Including the age variable in the model did not change the overall intervention effects ${ }^{6}$, demonstrating that both the Personal and Impersonal conditions effectively realigned perceived risk with actual risk for adults across the lifespan.

Next, we examined age effects. We found that the intervention produced immediate benefits for older and younger adults alike (Figure 1A, 1B; Figure 2A, 2B). Descriptive statistics for key variables by condition and age group are provided in Supplementary Table 1. We found that age (continuous variable) was not significantly related to change in perceived risk at Session $1(\beta=0.01,95 \%$ CI $[-0.08,0.10], t=0.27, p=.790)$, nor did age interact with prediction error $(\beta$ $=-0.04,95 \%$ CI $[-0.13,0.05], t=-0.95, p=.343$ ) or simulation condition (Unrelated vs.

Personal: $\beta=-0.06,95 \%$ CI $[-0.18,0.06], t=0.95, p=.340$, Impersonal vs. Unrelated: $\beta=-0.02$, $95 \%$ CI $[-0.14,0.11], t=-0.25, p=.803$, Personal vs. Impersonal: $\beta=0.08,95 \%$ CI [-0.05, $0.20], t=1.19, p=.236$ ). Overall, we found no significant age differences when perceived risk was assessed immediately after the intervention.

Next, we tested whether age was related to the longer-term effects of the interventions (Session 2). Using multiple linear regression, we predicted lasting change in perceived risk (delayed post-intervention - baseline) from the variables age (continuous), simulation condition, prediction error, and all interactions. We included a covariate for the duration of the delay period between sessions (ranging from 7-25 days). There was no significant main effect of age on lasting change in perceived risk at Session $2, \beta=0.02,95 \% \mathrm{CI}[-0.07,0.11], t=0.38, p=$ .704. However, there was an interaction between age and prediction error, such that effects of prediction error were not as evident in older adults after a delay, $\beta=-0.15,95 \%$ CI [-0.24, -0.06], $t=-3.30, p=.001$. In other words, numerical information about risk did not effectively induce longer-term learning in older adults (Figure 1C, 1D). 
a

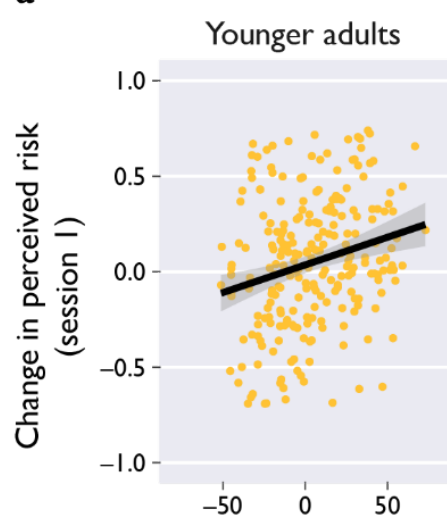

Middle-aged

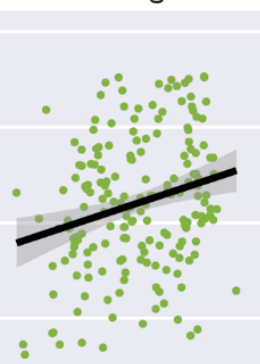

$-50 \quad 0 \quad 50$

Average prediction error

c

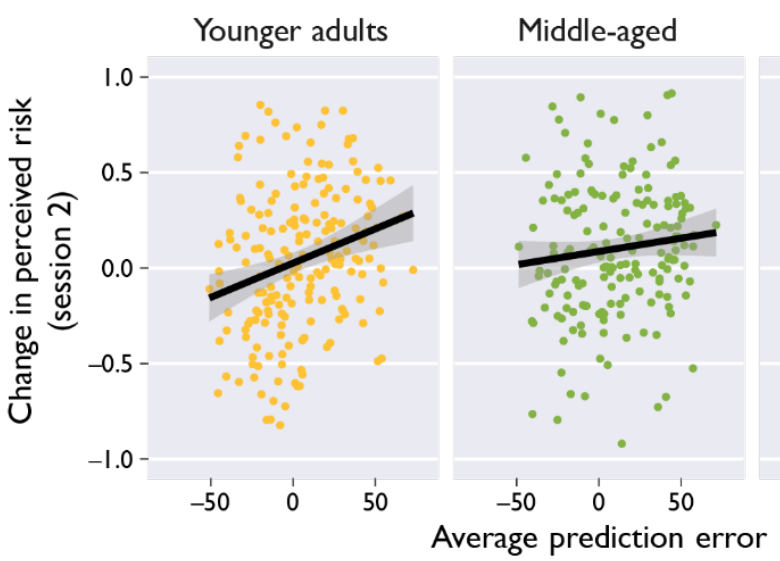

b

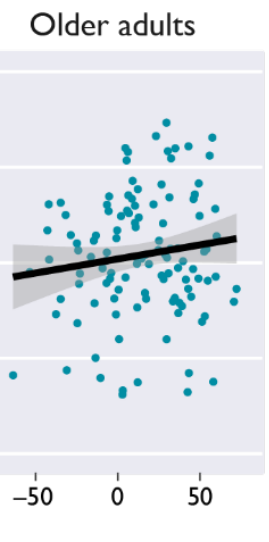

Older adults

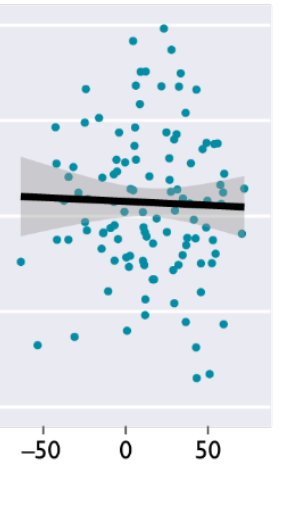

d
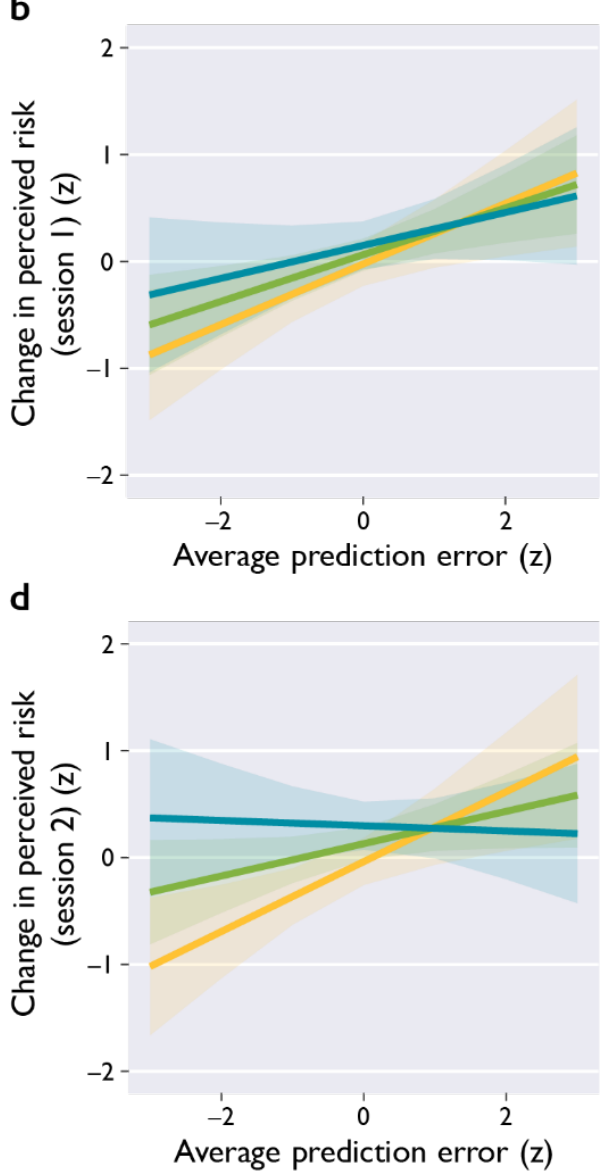

\begin{tabular}{|l|l|l|}
\hline Age group: & $18-39$ & $40-59$ \\
\hline
\end{tabular}

Figure 1. Prediction error drove change in perceived risk, but not for older adults.

A) During Session 1 (immediately post-intervention), average information prediction error scores were positively associated with change in perceived risk for Younger Adults (Pearson's $r(232)=0.24,95 \%$ CI $[0.11,0.36], p=.0002)$ and Middle-Aged Adults $(r(185)=0.24,95 \%$ CI $[0.11,0.38], p=.0007)$, but not Older Adults (Older Adults: $r(113)=0.16,95 \%$ CI [-0.02, 0.33], $p=.086$ ). B) Model-derived slope estimates (standardized variables) corresponding to the raw data depicted in panel A, indicating the main effect of prediction error after controlling for the effect of intervention condition. The interaction between prediction error and age (continuous) was non-significant, $\beta=-0.04,95 \% \mathrm{CI}[-0.13,0.05], t=-0.95, p=$ .343. C) During Session 2 (1-3 weeks post-intervention), prediction error was positively associated with change in perceived risk for Younger Adults $(r(202)=0.29,95 \% \mathrm{CI}[0.16,0.41], p=.00002)$ and Middle-Aged Adults $(r(172)=0.19,95 \%$ CI $[0.04,0.33], p=.012)$, but not Older Adults (Older Adults: $r(108)=-0.02,95 \%$ CI $[-0.21,0.16], p=.806)$. D) Model-derived slope estimates (standardized variables) corresponding to the raw data depicted in panel $\mathrm{C}$, indicating the main effect of prediction error after controlling for intervention condition and delay duration. There was an interaction between prediction error and age (continuous), $\beta=-0.15,95 \%$ CI $[-0.24,-0.06], t=-3.30, p=.001$. Overall Notes: Points in panels $\mathrm{A}$ and $\mathrm{C}$ depict subject scores (jittered for visualization), and lines depict correlations for each age group (two-sided tests, not corrected for multiple comparisons). Lines in panels $\mathrm{B}$ and $\mathrm{D}$ depict slopes derived from multiple linear regression models that included age as a continuous variable (two-sided omnibus tests). Error bands indicate 95\% confidence intervals. 
We also found an interaction between age and simulation condition, such that older adults reported a greater increase in perceived risk in the Personal simulation condition (Personal vs. Impersonal: $\beta=0.15,95 \%$ CI $[0.02,0.28], t=2.31, p=.021$, Personal vs. Unrelated: $\beta=0.13$, $95 \%$ CI [0.004, 0.25], $t=2.04, p=.042$, Impersonal vs. Unrelated: $\beta=-0.02,95 \%$ CI [-0.15, $0.10], t=-0.39, p=.700)$. Although this pattern of results is numerically consistent with the pattern in Session 1 (Figure 2A, 2B), the effect of the Personal simulation increasing perceived risk in older adults was enhanced over time (Figure 2C, 2D).

To further clarify age-related differences, we next compared our condition contrasts across age groups. We modified the Session 2 regression model described above to replace the continuous age variable with a categorical variable with three age bins (Younger Adults: 18-39, Middle-Aged: 40-59, Older Adults: 60-81). We found that relative to younger adults, older adults showed greater increases in perceived risk in the Personal condition than in the Impersonal condition ( $\beta=0.21,95 \%$ CI $[0.04,0.37], t=2.45, p=.015)$. Relative to younger adults, older adults also reported greater decreases in perceived risk in the Impersonal condition than in the Unrelated condition $(\beta=-0.18,95 \%$ CI $[-0.35,-0.01], t=-2.05, p=.041)$. The contrast between the Personal and Unrelated conditions did not differ between older adults and younger adults $(\beta$ $=-0.03,95 \%$ CI $[-0.19,0.14], t=-0.35, p=.724)$. No contrasts with the middle-aged group were statistically significant. Overall, we found that the Personal simulation condition elicited the greatest long-lasting increases in perceived risk for older adults. 
a

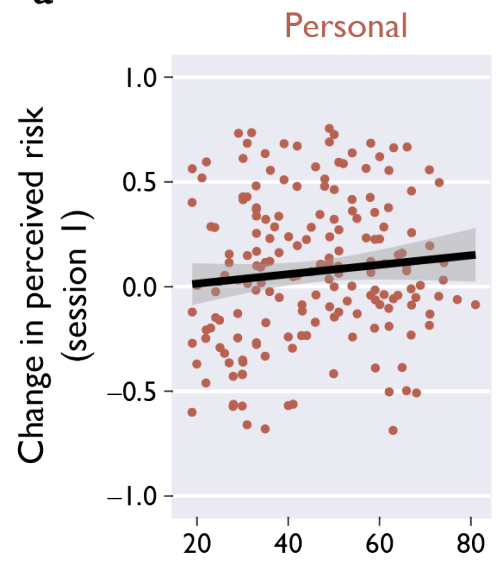

C

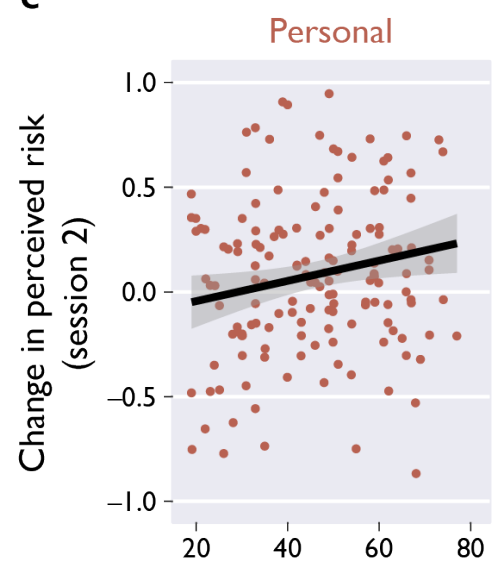

Impersonal

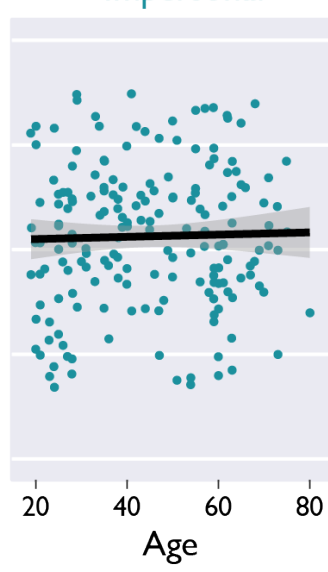

Impersonal

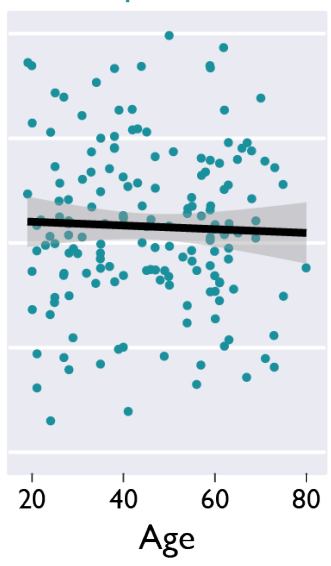

Unrelated

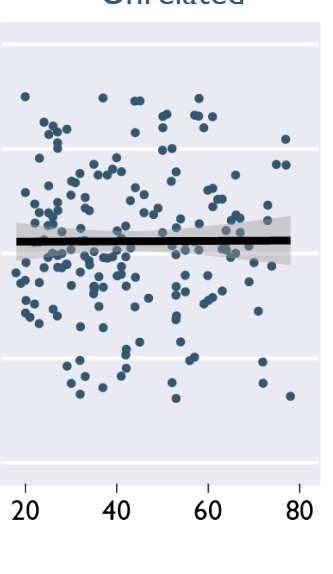

Unrelated

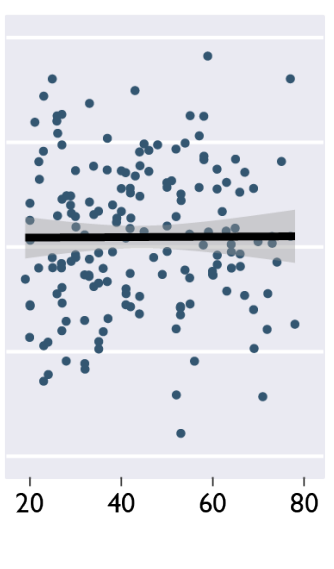

b

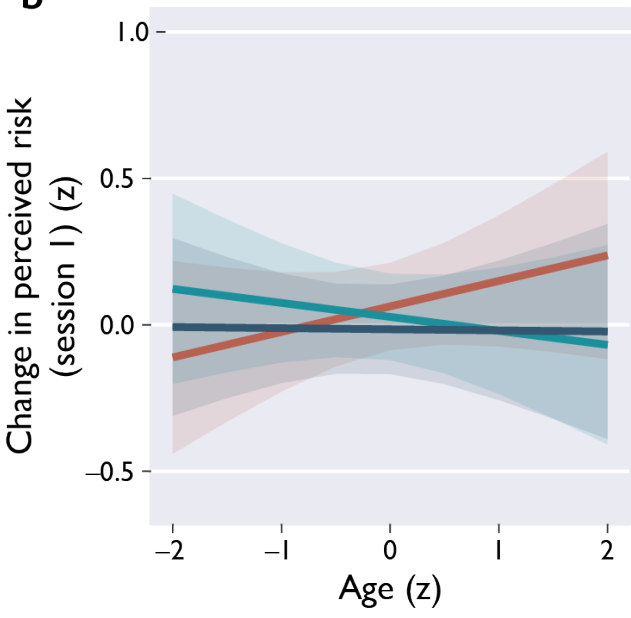

d

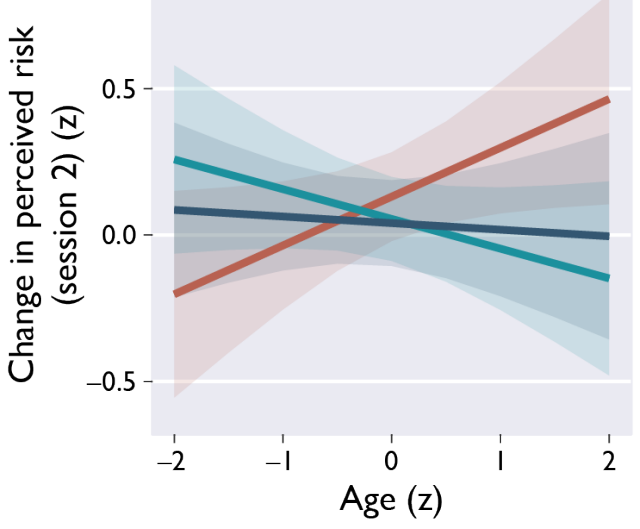

Simulation condition:

Personal

Impersonal

Unrelated

Figure 2. Comparing the effects of episodic simulations (Personal, Impersonal, and Unrelated) on perceived risk across the adult lifespan. A) During Session 1 (immediately post-intervention), there were no statistically significant associations between age and change in perceived risk (Personal: $r(179)=0.10$, 95\% CI [-0.05, 0.24], $p=.175$; Impersonal: $r(178)=0.03,95 \%$ CI [-0.12, 0.17], $p=.739$; Unrelated: $r(182)=0.004,95 \%$ CI [-0.14, 0.15], $p=.953)$. B) Model-derived slope estimates (standardized variables) corresponding to the raw data depicted in panel A, indicating the main effect of simulation condition after controlling for prediction error. The interaction between simulation condition and age (continuous) was non-significant $\left(\mathrm{F}_{(2,524)}=0.79, p=.455\right)$. C) During Session $2(1-3$ weeks postintervention), there was a positive association between age and change in perceived risk, selectively in the Personal simulation condition (Personal: $r(156)=0.16,95 \%$ CI $[0.003,0.31], p=.045$; Impersonal: $r(163)=-0.03,95 \%$ CI [-0.18, 0.13], $p=.731$; Unrelated: $r(169)=-0.04,95 \%$ CI $[-0.19,0.11], p=.608)$. D) Model-derived slope estimates (standardized variables) corresponding to the raw data depicted in panel $\mathrm{C}$, indicating the main effect of simulation condition after controlling for prediction error and delay duration. There was an interaction between simulation condition and age (continuous) $\left(\mathrm{F}_{(2,475)}=3.41, p=\right.$ .034). Overall Notes: Points in panels A and C depict subject scores (jittered for visualization), and lines depict correlations for each condition (two-sided tests, not corrected for multiple comparisons). Lines in panels B and D depict slopes derived from multiple linear regression models (two-sided omnibus tests).

Error bands indicate $95 \%$ confidence intervals. 
We hypothesized that the benefit of the Personal simulation for older adults may be enhanced after a delay because this condition could motivate individuals to independently seek out further information about local risk levels. To test this idea, we conducted an exploratory analysis in which we predicted post-intervention change in seeking information about local COVID-19 statistics (Session 2) from the variables age (continuous), simulation condition, prediction error, all relevant interaction terms, and the covariate for delay duration. There was an interaction between age and simulation condition predicting change in information-seeking, such that older adults selectively increased information-seeking during the weeks following the Personal simulation (Personal vs. Impersonal: $\beta=0.25,95 \%$ CI $[0.11,0.38], t=3.61, p<.001$, Unrelated vs. Personal: $\beta=-0.14,95 \%$ CI $[-0.27,-0.01], t=-2.18, p=.030$, Impersonal vs. Unrelated: $\beta=-0.10,95 \%$ CI $[-0.23,0.02], t=-1.59, p=.112)$. Overall, for older adults the Personal simulation was associated with increased information-seeking about local risk levels (Figure 3A, 3B), and longer-term increases in perceived risk (Figure 2C, 2D).

a

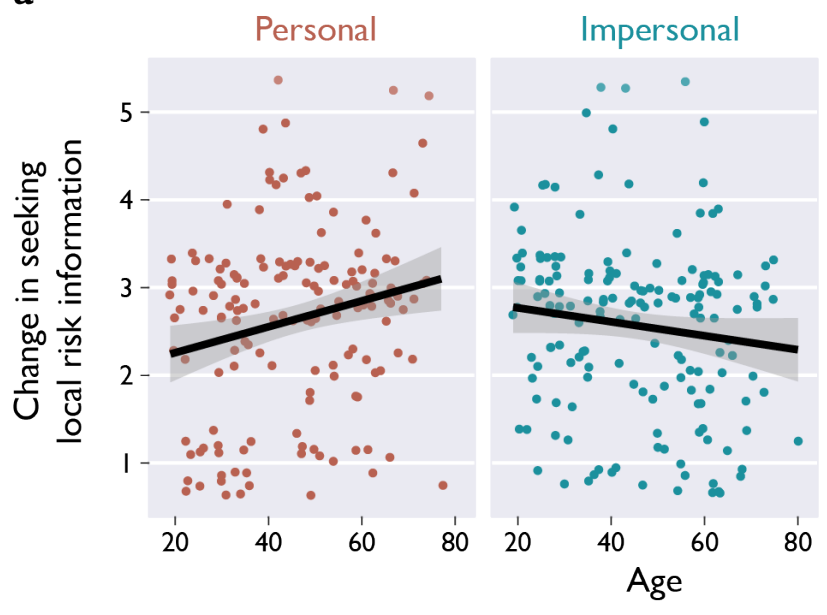

b

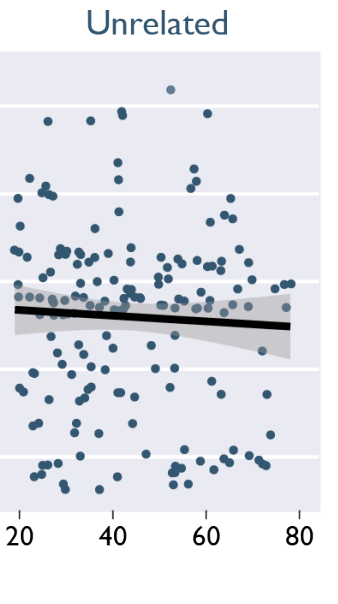

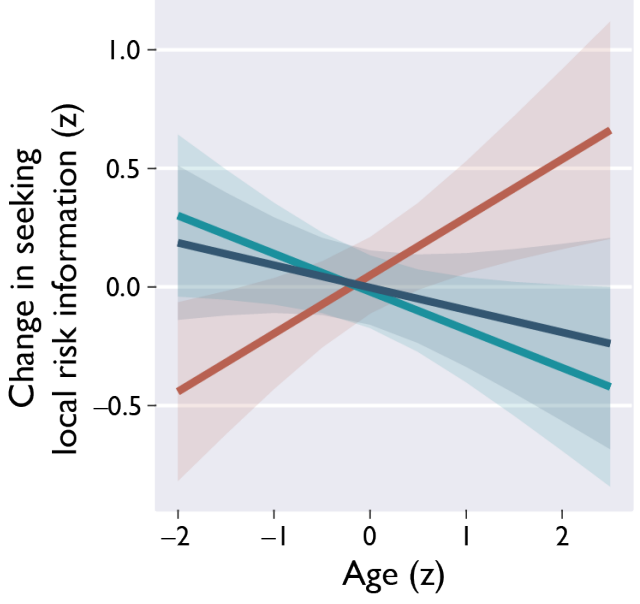

Simulation condition:

Personal

Impersonal

Unrelated

Figure 3. Testing the effects of age and episodic simulation (Personal, Impersonal, and Unrelated) on change in information seeking about local COVID-19 risk. A) Older adults in the Personal simulation condition reported increases in independent information-seeking about local risk statistics during the postintervention delay period (Personal: $r(156)=0.22,95 \%$ CI $[0.07,0.36], p=.006$; Impersonal: $r(163)=-$ $0.11,95 \%$ CI [-0.26, 0.04], $p=.149$; Unrelated: $r(169)=-0.06,95 \%$ CI [-0.21, 0.09], $p=.441)$. B) Model-derived slope estimates (standardized variables) corresponding to the raw data depicted in panel A, depicting the effect of age on change in information-seeking after controlling for prediction error and delay duration. There was an interaction between simulation condition and age (continuous) $\left(\mathrm{F}_{(2,475)}=\right.$ 5.92, $p=.003$ ). Overall Notes: Points depict subject scores (jittered for visualization). Lines depict correlations for each condition (two-sided tests, not corrected for multiple comparisons). Error bands indicate $95 \%$ confidence intervals. 
The COVID-19 pandemic has presented staggering new social and health-related challenges. In particular, older adults have been disproportionately impacted by the pandemic: Older adults are at substantially greater risk of severe illness, hospitalization, and death due to COVID-19 ${ }^{3}$. Compounding these health concerns, older adults may prioritize information differently than younger adults when considering health-related risk information ${ }^{12,20,21,36}$, and older adults are more susceptible to misinformation ${ }^{7-9}$. In this high-stakes context, it is crucial to develop interventions that convey information about health risks in a manner that is tailored to the needs of older adults.

Here, we investigated the age-related effects (both immediate and longer-term) of several strategies for conveying information about risk. Our novel informational intervention was effective for both older and younger adults alike ${ }^{6}$. Immediately after the intervention, older adults reported changes in perceived risk that were comparable to those reported by younger adults. However, age differences emerged over time: Although younger adults successfully retained learning after a delay of 1-3 weeks, older adults were more likely to lose the benefits of the intervention over time if the information was poorly matched to their emotional and cognitive processing characteristics. Here, we showed that numerical information about risk (quantified as information prediction errors) effectively drove longer-term learning in younger adults, but not older adults. This is consistent with prior evidence that, relative to younger adults, older adults learn more slowly from prediction errors during reinforcement learning tasks ${ }^{37,38}$. Crucially, older adults reported greater long-lasting increases in perceived risk only when they imagined the possible outcomes of risky decisions that affected themselves and close others. Imagining an impersonal or unrelated scenario did not increase perceived risk in older adults, either immediately or after a delay.

In an additional exploratory analysis, we also found that for older adults only, the personalized episodic simulation was associated with increased information seeking. During the post-intervention delay period (1-3 weeks), older adults (but not younger adults) who received the personalized simulation reported actively consuming more information about local COVID19 risk levels, relative to their pre-intervention habits. This finding suggests that the personalized episodic simulation helped motivate ongoing learning and cultivate a habit of information seeking. Recent research has shown that older adults tend to be less willing to seek new information, even deliberately choosing ignorance when the information could be negative ${ }^{22}$. Our intervention offers a promising new method to encourage information seeking in older adults. Overall, our results suggest that including a personalized imagination exercise can enhance the efficacy of interventions that target older adults, facilitating longer-term learning and better health-related decision making.

We found that the effect of numerical risk information on older adults was weakened over time, but the personalized imagination exercise elicited lasting increases in perceived risk and information-seeking. Older adults may be more prone to forgetting numerical risk information. However, another possibility is that they could have replaced or updated this 
knowledge with new information that was encountered after the intervention. We tested this account by comparing risk estimation accuracy during Session 2, but did not find evidence that older adults who engaged in more information-seeking became more accurate at estimating updated risk levels, regardless of the intervention condition (Supplementary Information, Session 2 Risk Estimation Accuracy). Overall, our results support the idea that older adults are more likely to forget numerical risk information, but personalized elements can elicit long-term intervention effects.

Taken together, our results suggest that certain strategies are more effective for inducing longer-term increases in perceived risk for older adults. Although older adults may be more prone to forgetting numerical information, a personalized episodic simulation may enhance both learning retention and information-seeking behaviors over time. Overall, both of these mechanisms may contribute to the beneficial effects of our intervention. Our results are generally consistent with the fundamental tenets of Socioemotional Selectivity Theory, which posits that older adults are more motivated to reinforce social connections and seek information that is personally-relevant or emotionally meaningful ${ }^{11,12,25}$. Imagining a personalized scenario that connects information with existing semantic and episodic memories may be an effective way to make risk information more memorable for older adults. Personalized interventions situate risk information in context, drawing on social connections to enhance salience. Our results also align with prior studies on episodic simulation, which have shown that imagining future scenarios can influence decision-making ${ }^{28}$, self-regulation ${ }^{30,31}$, and likelihood judgments ${ }^{34,35}$. Episodic simulations are most effective when they are vivid ${ }^{31}$, self-relevant ${ }^{33}$, emotional ${ }^{39}$, or repeatedly imagined $^{39}$; personalized interventions can target all of these elements. Our findings bridge theoretical insights from these two literatures to show that older adults are particularly responsive to personalized episodic simulations.

Throughout the course of the COVID-19 pandemic, Americans have underestimated the risk of engaging in many different everyday activities ${ }^{6}$. On average, our personalized intervention encouraged older adults to be more risk averse, reporting greater subjective perceived risk of engaging in various everyday activities (e.g., dining in a restaurant). In the context of the COVID-19 pandemic, instilling caution and risk-averse attitudes offers clear benefits for public health, especially for at-risk groups like older adults. However, for younger adults, an overall increase in risk-aversion (regardless of actual local risk levels) may not be a desirable outcome. We also found that our intervention bidirectionally improved the accuracy of risk-related beliefs in adults across the lifespan. The Personal and Impersonal simulation conditions were both effective at realigning perceived risk with actual risk, successfully mitigating risk underestimation and overestimation. However, the personalized intervention tended to increase perceived risk in older adults, regardless of their baseline misestimation bias. Overall, the findings reported here demonstrate that different intervention strategies may be needed to meet the needs of older adults: an intervention that takes into account cognition, 
motivation, and risk tolerance can encourage caution in older adults who are at higher risk of serious health outcomes.

Here, we measured subjective perceived risk of viral transmission associated with various everyday activities. Although this approach offers generalized insight into risk perception in everyday settings, there are several limitations. The true risk levels of these everyday scenarios are not known, and can vary widely depending on the circumstances. Ventilation, mask wearing, sanitation, crowding, and vaccination rate all contribute to the true risk level of a situation. Additionally, an individual's risk factors (e.g., pre-existing health conditions, age, vaccination status) also influence perceived risk. Overall, our measures of subjective perceived risk and prevalence-based exposure risk are useful heuristics for communicating the risk of virus exposure, but the exact likelihood of viral transmission (and the severity of subsequent illness) depend on the circumstances and the participants involved.

Although we conducted our study in the context of the COVID-19 pandemic, our findings may be broadly relevant to other health-related challenges. For example, annual influenza outbreaks pose a recurring health risk for older adults. Relative to their younger counterparts, older adults are far more likely to experience severe health complications due to the seasonal flu, and they are far more likely to die because of it ${ }^{40}$. The seasonal flu vaccination is a readily available and effective means of reducing health-related complications and death in older adults. Personalized episodic simulations that target risk beliefs about the seasonal flu might encourage older adults to get the vaccine each year. For example, messages promoting the flu vaccine could prompt individuals to imagine experiencing illness vs. wellbeing for themselves and close others. Incorporating personalized and socially-relevant elements could also improve communication of information about other health-related decisions for older adults (e.g., regarding lifestyle changes or medical procedures). Healthcare providers and policymakers could emphasize practical and personalized messaging to communicate information about risks to older adults. Future research can further explore these possibilities to apply episodic simulation to improve other health-related outcomes.

Acknowledgements: The study was funded by discretionary funding from the Duke Trinity College of Arts and Sciences to GSL and a U.S. National Institute on Aging grant (R01-AG058574) awarded to GSL and RC. AS is supported by a Graduate Research Fellowship from the National Science Foundation, and a Postgraduate Scholarship from the Natural Sciences and Engineering Research Council of Canada. Thanks to Aroon Chande, Seolha Lee, Quan Nguyen, Stephen J Beckett, Troy Hilley, Clio Andris, and Joshua S Weitz at Georgia Tech and Mallory Harris at Stanford for openly sharing the tools they developed to assess local virus levels, which made the present studies possible.

Author Contributions: AS, SH, MS, and GSL designed the studies. AS, SH, and MS created stimuli and survey materials. AS performed data collection. AS analyzed data with input from SH, MS, RAA, RC, and GSL. AS and MS drafted the paper, with input from SH, RAA, RC, and GSL. All authors approved of the final version.

Competing Interests Statement: The authors have no competing interests to report. 


\section{Methods}

This study is part of a larger project on risk perception during the COVID-19 pandemic. Other results from this larger project have been reported elsewhere ${ }^{6}$. The study was approved by the Duke University Health System IRB (Protocol \#00101720). Participants provided informed consent by reading a description of the online study and clicking a button that affirmed agreement. The design of the intervention was pre-registered, and age-related analyses were included under planned exploratory analyses (https://osf.io/6fjdy).

\section{Data Availability}

Raw and cleaned data are provided online via the Open Science Framework (https://osf.io/35us2/) ${ }^{41}$. Open-ended written responses to the episodic simulation have been omitted from the raw data to protect participant privacy, because personalized scenarios may include identifiable data. The full set of written responses from the episodic simulation task can be provided upon reasonable request, with IRB approval.

\section{Code Availability}

Statistical analyses were conducted using multiple linear regression in R (v4.0.3), implemented with R Studio (v1.3.1093). All scripts are provided online via the Open Science Framework (https://osf.io/35us $2 /)^{41}$. These scripts reproduce all data cleaning procedures, analyses, and plots used to generate the results reported in the manuscript.

\section{Participants}

We recruited a nationally-representative sample of 816 current U.S. residents (stratified by age, gender, and race to approximate the demographic makeup of the nation). Participants were recruited via Prolific, an online testing platform. Prolific curates nationally-representative samples by inviting select participants to complete the study, depending on their demographic characteristics. The demographic characteristics of the total sample recruited by Prolific were as follows: $48.7 \%$ men, $51.3 \%$ women; $14.3 \%$ between ages $18-27,18.9 \%$ between ages $28-37$, $16.4 \%$ between ages $38-47,16.6 \%$ between ages $48-57$, and $31.6 \%$ between ages $58-81 ; 74.4 \%$ white, $13.5 \%$ Black, 6.8\% Asian, 3.0\% mixed, 2.3\% other. We excluded 89 participants for the following preregistered reasons: missing COVID-19 statistics for their location (27), failing an attention check (27), providing off-topic or excessively short written responses to the Episodic Simulation task (e.g., answering a prompt for 2-3 sentences with only a few words) (34), or not providing age information (1). Additionally, 189 participants completed a control condition (Unguided Exploration) that was discussed in a separate report ${ }^{6}$ but was not relevant to the present analyses. The Unguided Exploration condition did not include the episodic simulation task or the risk estimation task. The primary goal of the present study was to compare the efficacy of the different simulation conditions across the adult lifespan, so the Unguided Exploration condition was not relevant for this research question. After these exclusions, the 
final sample consisted of 545 participants. Although age was included as a continuous variable in our primary statistical analyses, we binned participants into three age groups for a follow-up analysis and data visualization. The sample sizes of these age groups were as follows: Young Adults (ages 18-39, session $1 n=238$, session $2 n=205$ ), Middle-Aged (ages 40-59, session $1 n$ $=189$, session $2 n=176$ ), and Older Adults (ages 60-81, session $1 n=118$, session $2 n=113$ ).

\section{Procedure}

Survey. To assess subjective perceived risk, we asked participants to rate the riskiness (due to COVID-19) of engaging in 15 different activities in their local community, using a 5point Likert-type scale ( $1=$ Not at all risky, $5=$ Extremely risky). Activities included picking up takeout, grocery shopping (indoors, masked), exercising in a gym (indoors, no mask), dining in a restaurant (indoors, no mask), and going to a bar or club (indoors, no mask). We averaged ratings for the 15 items to calculate a composite score of perceived risk. Participants completed this subjective risk assessment three times: before the intervention, immediately after the intervention (Session 1), and 1-3 weeks after the intervention (Session 2). We calculated within-subjects change scores (post-intervention - baseline) for each testing session, to assess the effect of the intervention on perceived risk. To assess independent information-seeking, we also asked participants to report how much their COVID-related media consumption habits had changed during the post-intervention delay period. Participants rated change in information-seeking about local COVID-19 risk statistics on a 5-point Likert scale ( $1=$ Much less than usual, $5=$ Much more than usual).

Episodic Simulation Task. The Episodic Simulation task involved guided imagination through one of three scenarios that illustrated the potential consequences of risky decisions. During the simulation, participants were instructed to visualize events and details, then type responses in a text box. Participants were randomly assigned to one of three episodic simulation conditions in a between-subjects design: The Personal simulation (Session 1: $n=181$, Session 2: $n=158$ ), Impersonal simulation (Session 1: $n=180$, Session 2: $n=165$ ), or Unrelated simulation (Session 1: $n=184$, Session 2: $n=171$ ). In the Personal simulation, participants imagined themselves hosting a dinner party in their home, with four specific close others (e.g., friends or neighbors) as guests. Participants identified each guest by first name and/or relationship (e.g., "My sister Maria"), then visualized the guests and the setting (e.g., the dining room) in as much detail as possible. In this scenario, a guest began exhibiting symptoms of COVID-19 during dinner. The guest later confirmed a diagnosis and was hospitalized. The host then informed the other dinner party guests of the exposure, and eventually also became ill with COVID-19. The Impersonal simulation depicted a fictional character and his friends undergoing the same scenario. The Unrelated simulation described a scenario that was thematically related (a story about rabbits falling ill after eating rotten vegetables), but did not include any personalized or COVID-related elements. Full text for all simulation conditions is provided in the Supplementary Information (Episodic Simulation Text). 
Risk Estimation Task. After the Episodic Simulation, participants completed the Risk Estimation task, which involved estimating numerical risk levels in their local community. Participants received a brief tutorial about risk and probability, then were instructed to think about events of seven different sizes $(5,10,25,50,100,250$, and 500 people) that could happen in their location. For each event size, participants estimated the probability $(0 \%=$ Impossible ... $100 \%=$ Definitely) that at least one of the people attending the event was infected with COVID19. After estimating the risk levels for all event sizes, participants received veridical feedback about actual risk probabilities. Actual risk values were calculated based on the prevalence of active COVID-19 cases in each participant's county of residence ${ }^{42}$. We calculated information prediction error as a measure of misestimation, the average discrepancy between estimated and actual risk values across event sizes ${ }^{6}$.

\section{Statistics}

Statistical analyses were conducted using multiple linear regression. Continuous variables were standardized before submission to multiple linear regression. Factor variables for conditions were effect-coded. Visual inspection of histograms indicated that several variables exhibited high kurtosis, with some extreme values at both tails of the distribution. As a result, residuals from fitted models were larger for values at the tails. To correct for high kurtosis and meet the assumption of normality, we winsorized extreme values to the 5th and 95th percentiles. The variable for change in perceived risk (Session 1) was winsorized. As reported in detail elsewhere, winsorization improved model fits but did not change the statistical significance of our findings ${ }^{6}$. Additionally, we log-transformed the variable for actual risk (i.e., local case prevalence) to account for skew. Other variables were not transformed because distributions were approximately normal. Figures were produced using the ggplot $2^{43}$ (v3.3.2) and sjPlot ${ }^{44}$ (v2.8.6) packages. 


\section{References}

1. CDC. COVID Data Tracker. Centers for Disease Control and Prevention https://covid.cdc.gov/covid-data-tracker (2021).

2. Honein, M. A. Summary of Guidance for Public Health Strategies to Address High Levels of Community Transmission of SARS-CoV-2 and Related Deaths, December 2020. MMWR Morb. Mortal. Wkly. Rep. 69, (2020).

3. Chen, Y. et al. Aging in COVID-19: Vulnerability, immunity and intervention. Ageing Res. Rev. 65, 101205 (2021).

4. Stratton, C., Andersen, L., Proulx, L. \& Sirotich, E. When apathy is deadlier than COVID-19. Nat. Aging 1, 144-145 (2021).

5. Inouye, S. K. Creating an anti-ageist healthcare system to improve care for our current and future selves. Nat. Aging 1, 150-152 (2021).

6. Sinclair, A. H., Hakimi, S., Stanley, M., Adcock, R. A. \& Samanez-Larkin, G. Pairing Facts with Imagined Consequences Improves Pandemic-Related Risk Perception. PsyArXiv (2021) doi:10.31234/osf.io/53a9f.

7. Brashier, N. \& Schacter, D. Aging in an Era of Fake News. Curr. Dir. Psychol. Sci. 29, 316-323 (2020).

8. Grinberg, N., Joseph, K., Friedland, L., Swire-Thompson, B. \& Lazer, D. Fake news on Twitter during the 2016 U.S. presidential election. Science 363, 374-378 (2019).

9. Guess, A., Nagler, J. \& Tucker, J. Less than you think: Prevalence and predictors of fake news dissemination on Facebook. Sci. Adv. 5, eaau4586 (2019).

10. Pew Research Center. Share of U.S. adults using social media, including Facebook, is mostly unchanged since 2018. Pew Research Center https://www.pewresearch.org/fact-

tank/2019/04/10/share-of-u-s-adults-using-social-media-including-facebook-is-mostly-unchangedsince-2018/ (2019).

11. Carstensen, L. L., Fung, H. H. \& Charles, S. T. Socioemotional Selectivity Theory and the Regulation of Emotion in the Second Half of Life. Motiv. Emot. 27, 103-123 (2003).

12. Löckenhoff, C. E. \& Carstensen, L. L. Socioemotional Selectivity Theory, Aging, and Health: The Increasingly Delicate Balance Between Regulating Emotions and Making Tough Choices. J. Pers. 72, 1395-1424 (2004).

13. Jacklin, R., Sevdalis, N., Darzi, A. \& Vincent, C. A. Efficacy of cognitive feedback in improving operative risk estimation. Am. J. Surg. 197, 76-81 (2009).

14. Lermer, E., Streicher, B., Sachs, R., Raue, M. \& Frey, D. Thinking Concretely Increases the Perceived Likelihood of Risks: The Effect of Construal Level on Risk Estimation. Risk Anal. 36, 623-637 (2016).

15. Lustria, M. L. A. et al. A Meta-Analysis of Web-Delivered Tailored Health Behavior Change Interventions. J. Health Commun. 18, 1039-1069 (2013).

16. Torgerson, C. J., Porthouse, J. \& Brooks, G. A systematic review and meta-analysis of randomised controlled trials evaluating interventions in adult literacy and numeracy. J. Res. Read. 26, 234-255 (2003). 
17. Josef, A. K. et al. Stability and change in risk-taking propensity across the adult life span. J. Pers. Soc. Psychol. 111, 430-450 (2016).

18. Mamerow, L., Frey, R. \& Mata, R. Risk taking across the life span: A comparison of self-report and behavioral measures of risk taking. Psychol. Aging 31, 711-723 (2016).

19. Mata, R., Josef, A. K., Samanez-Larkin, G. R. \& Hertwig, R. Age differences in risky choice: a metaanalysis. Ann. N. Y. Acad. Sci. 1235, 18-29 (2011).

20. Meyer, B. J. F., Russo, C. \& Talbot, A. Discourse comprehension and problem solving: Decisions about the treatment of breast cancer by women across the life span. Psychol. Aging 10, 84-103 (1995).

21. Willis, S. L., Dolan, M. M. \& Bertrand, R. M. Problem solving on health-related tasks of daily living. in Processing of medical information in aging patients: Cognitive and human factors perspectives 199-219 (Lawrence Erlbaum Associates Publishers, 1999).

22. Hertwig, R., Woike, J. K. \& Schupp, J. Age differences in deliberate ignorance. Psychol. Aging (2021) doi:10.1037/pag0000603.

23. Eppinger, B., Hämmerer, D. \& Li, S.-C. Neuromodulation of reward-based learning and decision making in human aging. Ann. N. Y. Acad. Sci. 1235, 1-17 (2011).

24. Nassar, M. R. et al. Age differences in learning emerge from an insufficient representation of uncertainty in older adults. Nat. Commun. 7, 11609 (2016).

25. Carstensen, L. L. Social and emotional patterns in adulthood: Support for socioemotional selectivity theory. Psychol. Aging 7, 331-338 (1992).

26. Charles, S. T. \& Carstensen, L. L. Emotion Regulation and Aging. in Handbook of emotion regulation 307-327 (The Guilford Press, 2007).

27. Antonucci, T. C. \& Jackson, J. S. Social support, interpersonal efficacy, and health: A life course perspective. in Handbook of clinical gerontology 291-311 (Pergamon Press, 1987).

28. Rösch, S. A., Stramaccia, D. F. \& Benoit, R. G. Promoting farsighted decisions via episodic future thinking: A meta-analysis. PsyArXiv (2021) doi:10.31234/osf.io/53ju2.

29. Benoit, R. G., Paulus, P. C. \& Schacter, D. L. Forming attitudes via neural activity supporting affective episodic simulations. Nat. Commun. 10, 2215 (2019).

30. Gaesser, B. \& Schacter, D. L. Episodic simulation and episodic memory can increase intentions to help others. Proc. Natl. Acad. Sci. (2014) doi:10.1073/pnas.1402461111.

31. Peters, J. \& Büchel, C. Episodic Future Thinking Reduces Reward Delay Discounting through an Enhancement of Prefrontal-Mediotemporal Interactions. Neuron 66, 138-148 (2010).

32. Schacter, D. L., Addis, D. R. \& Buckner, R. L. Episodic simulation of future events: Concepts, data, and applications. in The year in cognitive neuroscience 2008 39-60 (Blackwell Publishing, 2008).

33. Dassen, F. C. M., Jansen, A., Nederkoorn, C. \& Houben, K. Focus on the future: Episodic future thinking reduces discount rate and snacking. Appetite 96, 327-332 (2016).

34. Gregory, W. L., Cialdini, R. B. \& Carpenter, K. M. Self-relevant scenarios as mediators of likelihood estimates and compliance: Does imagining make it so? J. Pers. Soc. Psychol. 43, 89-99 (1982). 
35. Sherman, S. J., Cialdini, R. B., Schwartzman, D. F. \& Reynolds, K. D. Imagining Can Heighten or Lower the Perceived Likelihood of Contracting a Disease: The Mediating Effect of Ease of Imagery. Pers. Soc. Psychol. Bull. 11, 118-127 (1985).

36. Samanez-Larkin, G. R. \& Knutson, B. Decision making in the ageing brain: Changes in affective and motivational circuits. Nat. Rev. Neurosci. 16, 278-289 (2015).

37. Eppinger, B., Schuck, N. W., Nystrom, L. E. \& Cohen, J. D. Reduced Striatal Responses to Reward Prediction Errors in Older Compared with Younger Adults. J. Neurosci. 33, 9905-9912 (2013).

38. Samanez-Larkin, G. R., Worthy, D. A., Mata, R., McClure, S. M. \& Knutson, B. Adult age differences in frontostriatal representation of prediction error but not reward outcome. Cogn. Affect. Behav. Neurosci. 14, 672-682 (2014).

39. Szpunar, K. K. \& Schacter, D. L. Get real: effects of repeated simulation and emotion on the perceived plausibility of future experiences. J. Exp. Psychol. Gen. 142, 323-327 (2013).

40. Monto, A. S. et al. Influenza control in the 21 st century: Optimizing protection of older adults. Vaccine 27, 5043-5053 (2009).

41. Sinclair, A. H., Hakimi, S., Stanley, M. L., Adcock, R. A. \& Samanez-Larkin, G. R. Pairing Facts with Imagined Consequences Improves Pandemic-Related Risk Perception. Open Science Framework, https://doi.org/10.17605/OSF.IO/35US2 (2020).

42. Chande, A. et al. Real-time, interactive website for US-county-level COVID-19 event risk assessment. Nat. Hum. Behav. 1-7 (2020) doi:10.1038/s41562-020-01000-9.

43. Wickham, H. ggplot2: Elegant Graphics for Data Analysis. (Springer-Verlag New York, 2016).

44. Lüdecke, D. sjPlot: Data Visualization for Statistics in Social Science. (2021). 